\title{
CYANATE ION IN COMPACT AMORPHOUS WATER ICE
}

\author{
Belén Maté ${ }^{1}$, Victor J. Herrero ${ }^{1}$, Yamilet Rodríguez-Lazcano ${ }^{1}$, Delia Fernández-Torre ${ }^{2}$, \\ Miguel A. Moreno ${ }^{1}$, Pedro C. Gómez ${ }^{3}$, and Rafael Escribano ${ }^{1}$ \\ ${ }^{1}$ Instituto de Estructura de la Materia, IEM-CSIC, Serrano 121, E-28006 Madrid, Spain \\ ${ }^{2}$ Departamento de Física Teórica de la Materia Condensada, Universidad Autónoma de Madrid, E-28050 Madrid, Spain \\ ${ }^{3}$ Departamento de Química Física I, Universidad Complutense, Unidad Asociada UCM-CSIC, E-28040 Madrid, Spain \\ Received 2012 July 16; accepted 2012 September 10; published 2012 October 22
}

\begin{abstract}
The $4.62 \mu \mathrm{m}$ infrared $\left(2164.5 \mathrm{~cm}^{-1}\right)$ absorption band, observed in ice mantels toward many young stellar objects, has been mostly attributed to the $v_{3}\left(\mathrm{CN}\right.$ stretch) band of $\mathrm{OCN}^{-}$ions. We present in this work a spectroscopic study of $\mathrm{OCN}^{-}$ions embedded in compact amorphous ice in a range of concentrations and temperatures relevant to astronomical observations together with quantum mechanical calculations of the $v_{3}$ band of $\mathrm{OCN}^{-}$in various $\mathrm{H}_{2} \mathrm{O}$ environments. The ice samples containing the ions are prepared through hyperquenching of liquid droplets of $\mathrm{K}^{+} \mathrm{OCN}^{-}$solutions on a substrate at $14 \mathrm{~K}$. The $\nu_{3} \mathrm{OCN}^{-}$band appears as a broad feature peaking at $4.64 \mu \mathrm{m}$ with a secondary maximum at $4.54 \mu \mathrm{m}$ and is much weaker than the corresponding peak in the liquid solution or in the solid salt. A similar weakening is observed for other $\mathrm{OCN}^{-}$absorption peaks at $7.66 \mu \mathrm{m}\left(2 \nu_{2}\right)$ and $8.20 \mu \mathrm{m}$ $\left(v_{1}\right)$. The theoretical calculations for the $v_{3}$ vibration lead to a range of frequencies spanning the experimentally observed width. This frequency spread could help explain the pronounced drop in the band intensity in the ice. The $\mathrm{OCN}^{-} v_{3}$ band in the present compact ices is also broader and much weaker than that reported in the literature for $\mathrm{OCN}^{-}$ions obtained by variously processing porous ice samples containing suitable neutral precursors. The results of this study indicate that the astronomical detection of $\mathrm{OCN}^{-}$in ice mantels could be significantly impaired if the ion is embedded in a compact water network.
\end{abstract}

Key words: infrared: general - line: identification - methods: laboratory

Online-only material: color figure

\section{INTRODUCTION}

The $4.62 \mu \mathrm{m}\left(2164.5 \mathrm{~cm}^{-1}\right)$ "XCN" band has been detected in many astrophysical objects and has promoted many arguments and investigations. It was first identified by Soifer et al. (1979) from the ground near the massive protostar W33A and later in many other sources, mainly high-mass young stellar objects (YSOs) detected from the ground (Pendleton et al. 1999; Pontoppidan et al. 2003) or from space (Gibb et al. 2000; Whittet et al. 2001). Most of these sources are embedded in dark clouds and are adequate for studying ice mantle chemistry in low-mass star-forming environments. The carrier of the $4.62 \mu \mathrm{m}$ feature is not uniquely assigned and, for this reason, it is commonly referred to in the astrophysical literature as "XCN", where X is an unknown, and where the $\mathrm{C} \equiv \mathrm{N}$ stretching vibration is assigned to the $4.62 \mu \mathrm{m}$ absorption. The presence of these $\mathrm{CN}$-bearing species is an observational indication of the rich chemistry that takes place in the ices. A statistical analysis of the center position of 39 YSOs in the observed band led van Broekhuizen et al. (2005) to conclude that the XCN absorption contains two components, one centered at $2165.7 \mathrm{~cm}^{-1}$ and the other at $2175 \mathrm{~cm}^{-1}$.

Many laboratory studies on astrophysical ice analogs have been conducted with the aim of identifying the carriers of the detected XCN feature. Grim \& Greenberg (1987) were the first to propose the cyanate $\left(\mathrm{O}-\mathrm{C} \equiv \mathrm{N}^{-}\right)$ion as a candidate, a species that could be formed easily in the laboratory by UV processing of CO: $\mathrm{NH}_{3}$ ice analogs. Different papers proved that the carrier of the laboratory feature was the $\mathrm{OCN}^{-}$ion (Schutte \& Greenberg 1997; Bernstein et al. 2000). Later, this species was generated by UV irradiation (Demyk et al. 1998; van Broekhuizen et al. 2004), proton irradiation (Palumbo et al. 2000; Hudson et al. 2001), or thermal mediated solid state chemistry (Novozamsky et al. 2001; Raunier et al. 2003; van Broekhuizen et al. 2004; Theule et al. 2011; Mispelaer et al. 2012) of ices composed of different combinations of $\mathrm{H}_{2} \mathrm{O}, \mathrm{CO}, \mathrm{CH}_{3} \mathrm{OH}, \mathrm{HNCO}, \mathrm{NH}_{3}$, and $\mathrm{N}_{2}$ (the latter molecule only in experiments with proton irradiation, as discussed by Palumbo et al. 2000). Van Broekhuizen et al. (2005) presented a compilation of all the laboratory experiments conducted until their publication on the spectroscopy of the $\mathrm{CN}$ stretch of $\mathrm{OCN}^{-}$ in interstellar ice analogs. The frequency position of the band varied between $2150 \mathrm{~cm}^{-1}$ and $2173 \mathrm{~cm}^{-1}$ in the different experiments. They concluded that of the two components empirically detected, only the low frequency peak could be assigned to $\mathrm{OCN}^{-}$, since the higher frequency contribution fails to be reproduced in laboratory experiments. However, a recent work by Öberg et al. (2011), where a global picture of ice abundances in cloud cores and high-mass protostars is presented, assigns $\mathrm{OCN}^{-}$as the only carrier of the entire $\mathrm{XCN}$ band via its correlation to $\mathrm{CO}$ abundances. They explain the spectral profile of the band as due to the different environments that could surround $\mathrm{OCN}^{-}$, which could be polar and non-polar depending on the sources.

In general, all laboratory investigations of ions in astrophysical ice analogs start with mixtures of neutral precursors that are then energetically processed to produce the charged species. These experiments not only lead to the formation of the ions in the ice but also provide valuable information about the formation mechanisms in astrophysical environments. Nonetheless, the different reactions that take place in the ices sometimes make it difficult to assign all the species formed or to decipher the particular environment sensed by the ions that may affect their infrared features. In our laboratory we have taken a 
different approach based on the sudden freeze of liquid droplets of aqueous solutions containing the ions of interest. Due to the high rate of cooling of the water droplets the technique was called "hyperquenching" (HQ). The method allows one to introduce the desired ions directly into the ice sample, thus unambiguously studying their spectra embedded in a water ice matrix. A recent work by our group indicates that the amorphous water ice generated by the sudden freeze of liquid droplets is nonporous as opposed to the vapor-deposited ice mixtures of varying porosity that act as precursors in previous laboratory experiments (see Baragiola 2003 and Maté et al. 2012 for a more thorough discussion of porosity and its dependence on ice generation conditions).

The morphology of water ice in astrophysical objects is an open field of research. Water is assumed to be locked as amorphous ice on the surface of dust grains in dense interstellar clouds, and often on cold bodies in planetary systems, where crystalline ices are also found. The ice morphology on these objects could change depending on their history and particular properties. It is known that in the laboratory, random vapor deposition (VD) of water molecules at low temperature forms microporous amorphous solids. In space, porous water ice can be formed when water molecules are deposited on dust grains in molecular clouds (Baragiola 2003). By contrast, when water molecules are formed on the grain surface through the reaction of adsorbed species, a compact amorphous ice is expected to be generated (Oba et al. 2009; Accolla et al. 2011; Romanzin et al. 2011). It has been proven in the laboratory that amorphous water ice can be made compact by energetic processing with highenergy ions or atoms, a process that can reproduce the effect of cosmic-ray bombardment in astronomical ices (Palumbo 2006; Accolla et al. 2011). Compact ice is thus probably the most common form of ice in grain mantles. As the $\mathrm{XCN}$ feature has been observed in ice grains on molecular clouds, where a rich chemistry takes place activated by different energetic processes, there is a high probability of finding $\mathrm{OCN}^{-}$in a compact water ice environment. As indicated above, previous laboratory studies have used astronomically realistic methods (UV or ion bombardment, thermal processing) for the production of ions in (porous) vapor-deposited ice mixtures. The energetic processing implied in these types of experiments can induce simultaneous chemical and morphological changes and could even lead to a segregation of ionic networks within the ice sample (Gálvez et al. 2010). The approach taken in this work is different. It concentrates on isolated ions embedded in compact ices, where the ice morphology and the $\mathrm{OCN}^{-}$ion environment could mimic more closely the conditions in the presumably compact interstellar ice mantles, although the generation procedure is not astrophysically relevant. In any case, the results of the two types of experiments are complementary and should help us to interpret astronomical observations.

In this paper, we present a study of the infrared spectra of the cyanate ion $\left(\mathrm{OCN}^{-}\right)$embedded in compact ice water. To generate these ices we used the HQ technique in a similar way as that described in our previous papers (Maté et al. 2009; Gálvez et al. 2010), devoted to the $\mathrm{NH}_{4}{ }^{+}$and the $\mathrm{HCOO}^{-}$ions. We will examine the effect of the compact water ice matrix on the infrared spectrum of the $\mathrm{OCN}^{-}$ion in comparison with previous literature spectra of the ion in different laboratory ices. To support the experimental results, we have conducted ab initio calculations of model systems containing water and $\mathrm{OCN}^{-}$and we have predicted their infrared spectral frequencies in an attempt to unravel specific spectroscopic features.

\section{EXPERIMENTAL PROCEDURES}

Water ices containing the cyanate ion were generated through the sudden freezing of droplets of a diluted aqueous solution of KOCN on a cold Si substrate located inside a vacuum chamber. This HQ technique was developed by Mayer and coworkers (Mayer 1985; Johari et al. 1987), and allows the creation of ice samples of ions surrounded by water molecules (Maté et al. 2009; Gálvez et al. 2010). The general experimental setup was described in previous publications (Maté et al. 2003; Gálvez et al. 2008; Maté et al. 2012) and here we will focus only on the most relevant aspects. The cold $\mathrm{Si}$ substrate is mounted in close contact with the cold head of a closed cycle He cryostat (ARS 4HW) inside a high vacuum chamber. The substrate temperature can be controlled with $1 \mathrm{~K}$ accuracy between $14 \mathrm{~K}$ and $300 \mathrm{~K}$. The vacuum chamber is evacuated with a turbomolecular pump to a background pressure of $\sim 10^{-8} \mathrm{mbar}$ and it is coupled to a Bruker Vertex 70 FTIR spectrometer through a purged pathway. Normal incidence transmission spectra of the ice samples were recorded, with $2 \mathrm{~cm}^{-1}$ resolution, using an MCT detector refrigerated with liquid nitrogen.

A pulsed valve (General Valve, series 9) with a $0.5 \mathrm{~mm}$ nozzle exit, usually employed for the generation of free jets and molecular beams (Abad et al. 1995), was filled with a KOCN: $\mathrm{H}_{2} \mathrm{O}$ solution of 3:100 molecular ratio. The KOCN salt presented $100 \%$ dissociation in water solution at room temperature, releasing $\mathrm{OCN}^{-}$and $\mathrm{K}^{+}$ions. When the valve was actuated, liquid droplets were expelled and impinged on the cold $\mathrm{Si}$ substrate placed at $\sim 10 \mathrm{~mm}$ from the nozzle exit. Once the droplets hit the substrate, held at $14 \mathrm{~K}$, their cooling rate is very high and the segregation and/or ion-pairing that may occur is minimal (see Fleissner et al. 1995). After deposition, the valve was moved back by means of translational feed-trough and the substrate was rotated perpendicular to the infrared radiation in order to record the infrared transmission spectra.

The concentrations of the HQ-generated ices are slightly higher than those of the liquid solutions from which they originate. This salt enrichment is probably caused by a partial evaporation of water from the droplets during their flight to the substrate. This behavior has already been observed by other authors (Mayer 1985). The initial liquid solution concentration of 3:100 KOCN: $\mathrm{H}_{2} \mathrm{O}$ was increased in the solid deposits to a value between $3.5 \%$ and $6 \%$, depending on the experiment. Spectra of the initial liquid solutions were also recorded for reference in a separate cell at room temperature. The water concentration in the ice mixtures was estimated by reference to the $3200 \mathrm{~cm}^{-1}$ water band intensity value reported by Mastrapa et al. (2009) at $15 \mathrm{~K}, A\left(\mathrm{H}_{2} \mathrm{O}\right)=1.9 \times 10^{-16} \mathrm{~cm} \mathrm{~mol}^{-1}$. The concentration of the $\mathrm{OCN}^{-}$species was evaluated from the spectra of the deposits that remain after sublimation of water. To estimate the number of molecules we used the intensity of the $v_{3}$ band of $\mathrm{OCN}^{-}$at $\sim 2170 \mathrm{~cm}^{-1}$ given by van Broekhuizen et al. (2004), $A\left(\nu, \mathrm{OCN}^{-}\right)=1.3 \times 10^{-16} \mathrm{~cm} \mathrm{~mol}^{-1}$, for a processed $\mathrm{H}_{2} \mathrm{O} / \mathrm{NH}_{3} / \mathrm{HNCO}=120 / 10 / 1$ ice at $122 \mathrm{~K}$.

\section{THEORETICAL CALCULATIONS}

\subsection{Methodology}

To provide support to the interpretation of our experimental observations, we have carried out theoretical calculations of two kinds. On one side, we have studied molecular aggregates of the cyanate anion and one water molecule, at the density functional theory (DFT) level, with a B3LYP/aug-cc-pVDZ functional 
and basis set. We have searched for equilibrium structures, with well-defined minima in the potential energy surface, and based on these structures, we have calculated the infrared spectra. These calculations are performed using Gaussian03 (Frisch et al. 2004). On the other side, we have tried to simulate the environment of cyanate within a water network, by means of a number of possible structures obtained by substitution of two water molecules in an Ih ice solid by one $\mathrm{OCN}^{-}$anion and one $\mathrm{Na}^{+}$or $\mathrm{K}^{+}$cation (Maté et al. 2009). The cations are introduced to keep the neutral structure of the crystal, but are not expected to influence the results that concern the cyanate part. These calculations are done using the SIESTA suite of programs (Ordejón et al. 1996; Soler et al. 2002) which is especially adequate for solids. The substituted structures are relaxed and their IR spectra are predicted. For the SIESTA runs we have used DFT natural atomic orbitals (NAO) with $\mathrm{H}, \mathrm{O}$, and N pseudopotentials from Martín-Llorente et al. (2006), C and $\mathrm{K}$ pseudopotentials from the SIESTA Web page, and a new pseudopotential for $\mathrm{Na}$, optimized and tested specifically for this work.

The predictions for the fundamental vibrations of all these structures are listed in Table 1 (see below), together with a summary of the observed spectra. The main results of the theoretical calculations are given below.

\subsection{Cyanate-water Dimers}

We have found two different species of $\mathrm{OCN}^{-} \ldots\left(\mathrm{H}_{2} \mathrm{O}\right)$, depending on which end of the cyanate the water molecule is linked to, as represented in the upper panel of Figure 1.

The Gaussian calculations predict for the $\mathrm{C}-\mathrm{N}$ stretching band, $v_{3}$, values at $2227 \mathrm{~cm}^{-1}$ and $2206 \mathrm{~cm}^{-1}$, for the N...H and $\mathrm{O} \ldots \mathrm{H}$ dimers in the upper panel of Figure 1, left and right, respectively. A modest estimation of the anharmonicity of this vibration can be made by applying a 0.9748 factor (Ref: http://cccbdb.nist.gov/vsfcalc3.asp?method $=8 \&$ basis $=17)$ to these values, as indicated in Table 1 . The other two fundamentals, the $\mathrm{O}-\mathrm{C}$ stretching band, $v_{1}$, and the $\mathrm{OCN}^{-}$bending, $v_{2}$, are much weaker than $v_{3}$, and may have limited astrophysical relevance, in principle. In the experimental spectra, $v_{1}$ always appears as a doublet, as a consequence of a well-known, strong Fermi resonance with $2 v_{2}$, the first overtone of the bending vibration.

\subsection{Cyanate within Ice Network}

We have explored up to 28 different ways to insert the ionic cyanate species into a simple water ice unit cell by replacement of $\mathrm{H}_{2} \mathrm{O}$ molecules by $\mathrm{OCN}^{-}$and $\mathrm{Na}^{+}$or $\mathrm{K}^{+}$. We have studied concentrations of $6: 1$ for $\mathrm{H}_{2} \mathrm{O}: \mathrm{NaOCN}$, and $6: 1$ and 14:1 for $\mathrm{H}_{2} \mathrm{O}: \mathrm{KCN}$. The lower panel of Figure 1 presents schematic views of the lowest energy ice networks. The energetically most stable structures were selected to predict their corresponding infrared spectra. In general, the calculated frequencies spread over a region of $\sim 30$ to $40 \mathrm{~cm}^{-1}$ for each vibration. Interestingly, two sets of values are also predicted for the $\mathrm{C}-\mathrm{N}$ vibration, one in the $2170-2180 \mathrm{~cm}^{-1}$ region, and another at $2160 \mathrm{~cm}^{-1}$. These values correspond to structures in which N...H distances are shorter than O...H distances, in the first case, and the opposite in the second, in good concordance with the dimers calculations.

\section{RESULTS AND DISCUSSION}

Figure 2 displays the spectra of three phases of the cyanate ion as prepared in our experiments. Whereas the $\mathrm{OCN}^{-}$strongest (a) Dimers

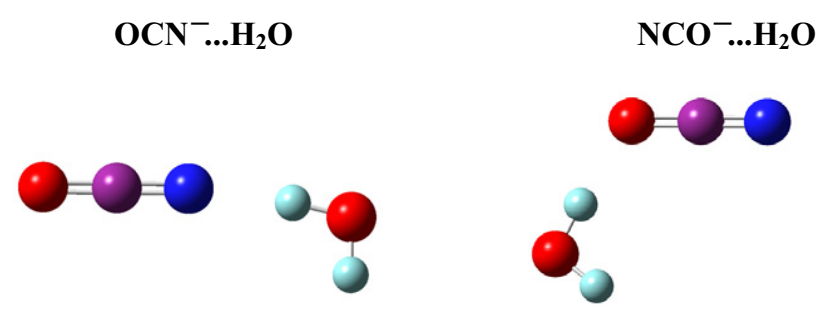

(b) Ice networks
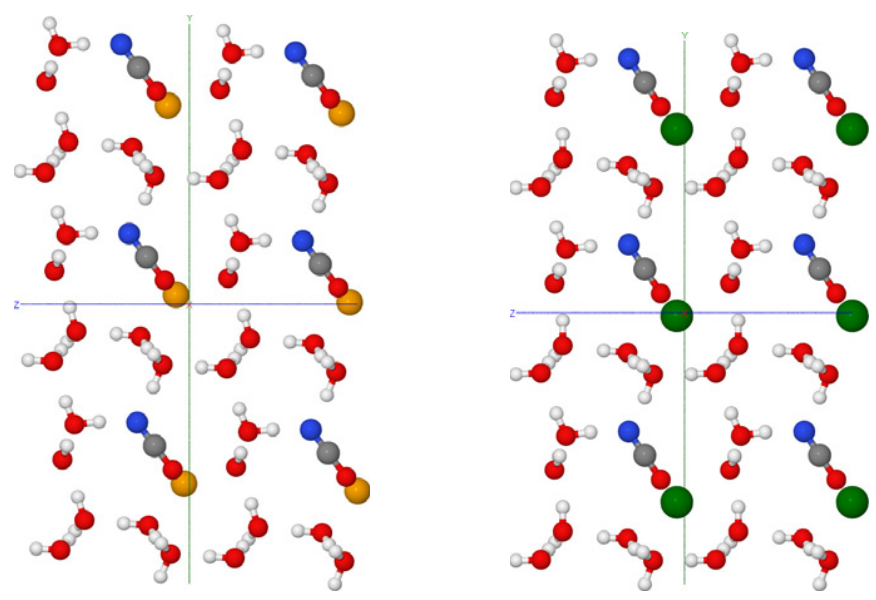

Figure 1. (a) Schematic representation of the equilibrium structures of $\mathrm{OCN}^{-} \ldots \mathrm{H}_{2} \mathrm{O}$ dimers calculated in this work. (b) Most stable structures of ice networks where $\mathrm{H}_{2} \mathrm{O}$ molecules have been replaced by $\mathrm{OCN}^{-}$and $\mathrm{Na}^{+}$ (left) and $\mathrm{OCN}^{-}$and $\mathrm{K}^{+}$(right).

(A color version of this figure is available in the online journal.)

peak, the $v_{3} \mathrm{CN}$ stretching band at $\sim 2200 \mathrm{~cm}^{-1}, \sim 4.55 \mu \mathrm{m}$, is clearly prominent in the spectra of the room temperature solution (top), it looks much weaker in that of the HQ deposit at $14 \mathrm{~K}$ (middle), to the point of wondering if the concentration had been drastically reduced in the process. However, when water is evaporated after heating to $210 \mathrm{~K}$, the spectrum of the cyanate ion is fully recovered (bottom), allowing a measurement of the amount of solid remaining. An equivalent behavior is appreciated for the $\mathrm{OCN}^{-} v_{1} / 2 v_{2}$ Fermi pair, at 1200 and $1300 \mathrm{~cm}^{-1}, 8.3$ and $7.7 \mu \mathrm{m}$, respectively. This effect bears resemblance to our observations for the ammonium and formate ions (Maté et al. 2009; Gálvez et al. 2010). In the former of these, the $v_{4}$ bending band of $\mathrm{NH}_{4}{ }^{+}$was apparently missing in the HQ sample, whereas in the latter we observed only a moderate intensity reduction for the formate bands.

The broadening and weakening of the cyanate vibrations can be rationalized in terms of the intrinsic disorder of the amorphous water ice structure created by the HQ technique. The sudden freeze of the liquid solution droplets when they impinge on the cold surface is believed to prevent the diffusion of the ions within the water. Hence, the cyanate ions remain in the ice matrix surrounded by water molecules, and now, in contrast to the homogeneous solvation sphere environment in the liquid phase, encounter different surroundings. The inhomogeneity in the arrangement of the water molecules that are first neighbors of cyanate ions may cause a broadening of the bands via a frequency spreading, as suggested by our SIESTA calculations, which show a range of predicted wavenumber values for this vibration, depending on the final relaxed structure 
Table 1

Main Vibrational Modes of $\mathrm{OCN}^{-}$

\begin{tabular}{|c|c|c|c|c|c|c|c|}
\hline \multirow[t]{2}{*}{ Mode } & \multicolumn{2}{|c|}{ HQ Deposit } & \multirow[t]{2}{*}{ Solid Residue } & \multicolumn{2}{|c|}{ Dimers $^{b}$} & \multicolumn{2}{|c|}{ Ice Network } \\
\hline & $\left(\mathrm{cm}^{-1}\right)$ & $(\mu \mathrm{m})^{\mathrm{a}}$ & & $\mathrm{OCN}^{-} \ldots \mathrm{H}_{2} \mathrm{O}$ & $\mathrm{H}_{2} \mathrm{O} \ldots \mathrm{OCN}^{-}$ & Range $^{c}$ & Range $^{\mathrm{d}}$ \\
\hline$v_{3}, v \mathrm{CN}$ & 2200,2156 & $4.545,4.638$ & 2183,2160 & 2171 & 2150 & $2180-2170$ & 2160 \\
\hline id, ${ }^{13} \mathrm{C}$ & 2114 & & 2113 & & & & \\
\hline $2 v_{2}$ & 1306 & 7.657 & 1300 & & & & \\
\hline$v_{1}, v \mathrm{OC}$ & 1220 & 8.197 & 1207 & 1244 & 1197 & $1250-1230$ & $1260-1250$ \\
\hline id, ${ }^{13} \mathrm{C}$ & & & 1191 & & & & \\
\hline$\nu_{2}, \alpha \mathrm{OCN}$ & hidden & & 628,639 & 608,606 & 610,597 & $605-570$ & $600-590$ \\
\hline
\end{tabular}

Notes. Column headings HQ Deposit and Solid Residue correspond to the experimental observations, and the rest to calculations, of the $\mathrm{OCN}^{-} \ldots \mathrm{H}_{2} \mathrm{O}$ clusters, and of solids obtained by $\mathrm{OCN}^{-}$replacement of $\mathrm{H}_{2} \mathrm{O}$ molecules. All values are in $\mathrm{cm}^{-1}$ except where indicated.

${ }^{\text {a }}$ Suggested values for astrophysical observation, in microns.

${ }^{\mathrm{b}}$ Anharmonicity corrected wavenumbers by a 0.9748 factor.

${ }^{c}$ Structures where average N. ..H distances are shorter than O. ..H distances.

d Opposite of footnote "c."

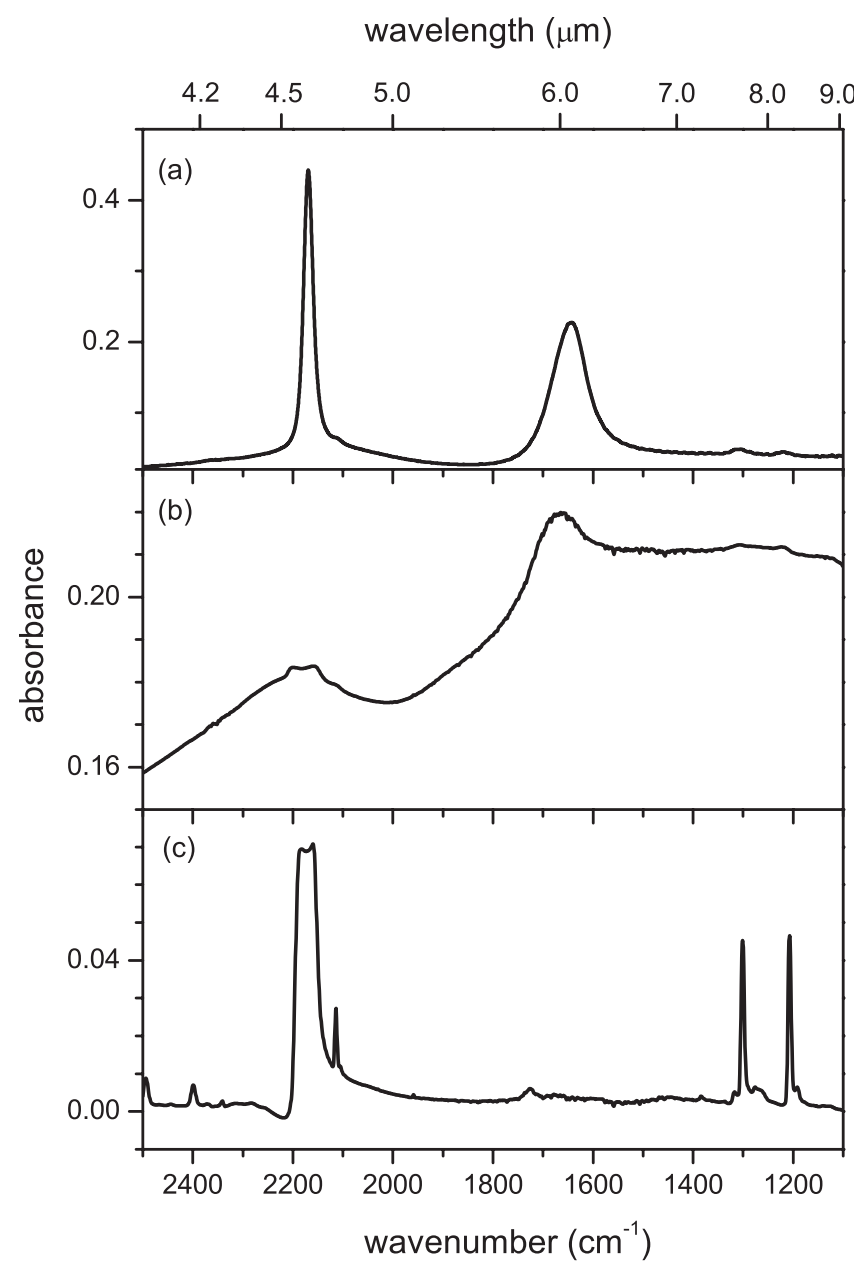

Figure 2. Transmission IR spectra of (a) (3:100) liquid solution of KOCN in water at ambient temperature, (b) solid generated by hyperquenching at $14 \mathrm{~K}$ of the solution presented in panel (a), and (c) solid residue obtained after heating the sample in panel (b) to $210 \mathrm{~K}$.

of the network. This spreading is evidenced in the spectrum as an apparent intensity decrease. We put forward a similar explanation in our previous paper on the ammonium ion (Maté et al. 2009), where the even higher intensity decrease of the bending mode of $\mathrm{NH}_{4}{ }^{+}$in compact ice suggested that the motion of the light hydrogen atoms was strongly affected by the surroundings.

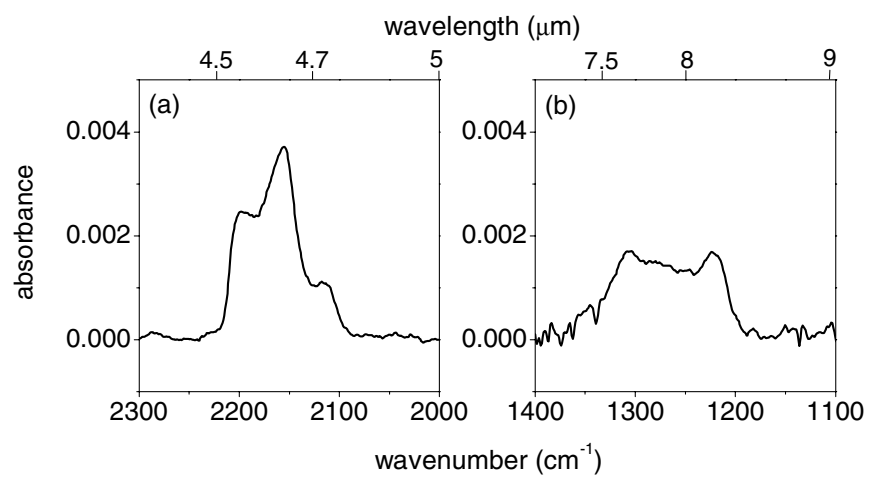

Figure 3. Enlargement of the spectral features presented in panel (b) of Figure 2 in the two spectral regions were the $\mathrm{OCN}^{-}$ion bands appear. The spectra in this figure have been obtained by subtracting a spectrum of pure $\mathrm{H}_{2} \mathrm{O}$ ice at the same temperature.

The lower panel of Figure 2 displays the spectrum of the residue remaining after water sublimation and molecular arrangement of the $\mathrm{OCN}^{-}$and $\mathrm{K}^{+}$ions to form $\mathrm{KOCN}$. The comparison of the integrated band intensity of the ionic bands in this residue with respect to that in the HQ matrix can provide a quantitative estimation of the attenuation suffered by the ion in the compact ice environment, which is valuable information for the observational community. This result is commented on below in Section 5 .

Figure 3 shows enlargements of the HQ spectra in panel (b) of Figure 2, in the two spectral regions where the main cyanate bands are present. A spectrum of pure water at the same temperature has been subtracted to highlight the band profiles of these absorptions. In the $\mathrm{CN}$ stretching region, panel (a), we can distinguish two features peaking at $\sim 2197$ and $2156 \mathrm{~cm}^{-1}$, plus a weaker third peak at $2114 \mathrm{~cm}^{-1}$ corresponding to the ${ }^{13} \mathrm{C}$ isotopomer. The interpretation of the doublet can be made based on our theoretical calculations for $\mathrm{OCN}^{-}\left(\mathrm{H}_{2} \mathrm{O}\right)$ aggregates, which predict two different values for this vibration, depending on which end of the cyanate ion the water molecule is linked to. According to the prediction, the high-frequency peak would correspond to the OCN-HOH species, whereas the lowfrequency band would be assigned to the $\mathrm{HOH}-\mathrm{OCN}$ structure. The maximum in the band intensity is associated with the latter structures. The splitting observed in the experimental spectrum is larger than that estimated in the theoretical calculations, but an 


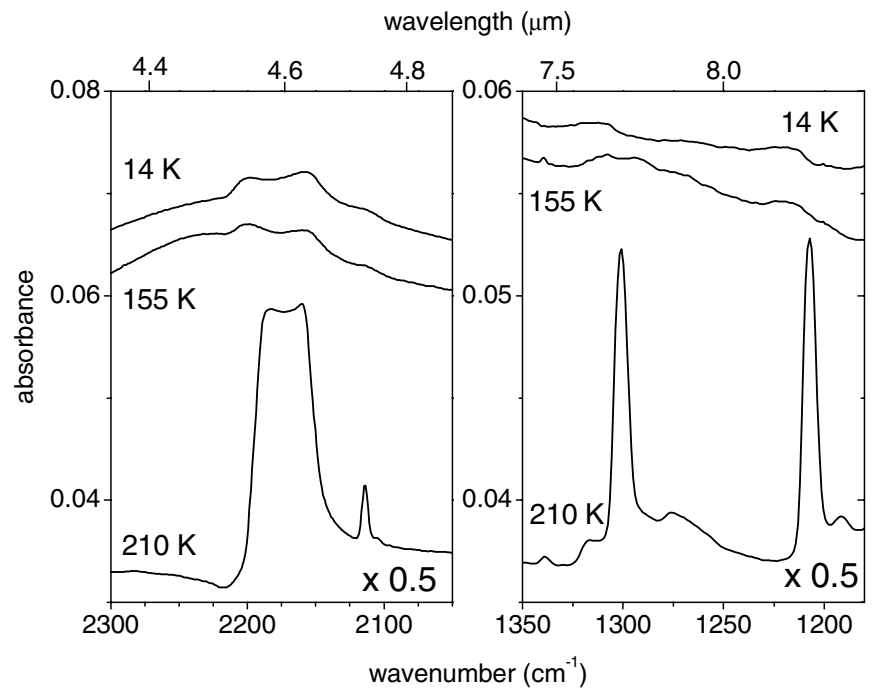

Figure 4. Thermal evolution of an $\mathrm{OCN}^{-} / \mathrm{K}^{+} / \mathrm{H}_{2} \mathrm{O}$ ice matrix obtained by hyperquenching of a (3:100) KOCN: $\mathrm{H}_{2} \mathrm{O}$ liquid solution. The ice was generated at $14 \mathrm{~K}$ and annealed to $155 \mathrm{~K}$, and then to $210 \mathrm{~K}$ to sublimate the water.

explanation based on the formation of a small number of stable dimers seems logical. Unfortunately, the present calculations do not provide a reliable estimate of the intensities for the various configurations and cannot be used to support this observation. The fact that the $v_{3}$ band is broader in the HQ samples and even has a secondary peak is probably related to the wider range of structures sampled by the generation procedure that freezes diverse $\mathrm{OCN}^{-}$solvation configurations from the original liquid droplets. In contrast, the processing of vapor-deposited mixtures provides sufficient energy to produce a rearrangement leading to a smaller set of (favorable) structures and thus to a narrower absorption band. In panel (b), in the $1400-1100 \mathrm{~cm}^{-1}$ region, two broad cyanate peaks at 1300 and $1200 \mathrm{~cm}^{-1}$ are visible and are assigned to the $2 v_{2} / v_{1}$ modes. The main vibrational bands observed and calculated for the $\mathrm{OCN}^{-}$species, including those measured for the ${ }^{13} \mathrm{C}$ isotopomer, are listed in Table 1 , where the features with a higher observational relevance are indicated in microns.

Figure 4 presents the thermal evolution of the compact ice matrix, starting from the HQ deposit at $14 \mathrm{~K}$. Water crystallization at $155 \mathrm{~K}$ was followed through the changes of shape and intensity of the main water bands in its IR spectrum (not shown in the figure). However, as can be appreciated in Figure 4 (left panel), the cyanate $v_{3}$ band does not change significantly during ice crystallization, similar to our observations for $\mathrm{NH}_{4}{ }^{+}$ embedded in compact water ice (see Figure 4 in Gálvez et al. 2010), and in contrast to its evolution in vapor-deposited ices, as reported in the literature (van Broekhuizen et al. 2005). The differences can be explained in terms of the dissimilar porous structure of both kinds of deposits. In particular, it is interesting to compare our results in Figure 4 with those presented by van Broekhuizen et al. (2004). In Figure 1 of that paper they showed the thermal evolution of a $(120: 10: 1) \mathrm{H}_{2} \mathrm{O}: \mathrm{NH}_{3}: \mathrm{HOCN}$ ice mixture generated by vapor deposition at $15 \mathrm{~K}$. Even at $15 \mathrm{~K}$ some $\mathrm{OCN}^{-}$ions were already present in the sample and a peak at $2170 \mathrm{~cm}^{-1}$, observed in the infrared spectrum of the ice, was assigned to this species. When the sample was warmed, the ion/acid fraction increased, and at $122 \mathrm{~K}$ all the isocyanic acid was transformed to cyanate. At all temperatures the $\mathrm{CN}$ stretch band of cyanate appears as a symmetric band at about $2170 \mathrm{~cm}^{-1}$ with $23 \mathrm{~cm}^{-1}$ FWHM. The band profile described in this experiment differs from the one we observe in our compact water ice matrix in Figure 4. Both experiments correspond to a diluted cyanate concentration of 1:120 in van Broekhuizen et al. (2004) and 3:100 in this work. A qualitative comparison can be carried out by using the intensity of, e.g., the $1600 \mathrm{~cm}^{-1}$ water band present in both spectra. The conclusion is that, beyond the slightly different concentrations, the $\mathrm{CN}$ stretch band appears weaker in HQ compact ice matrices than in VD porous ices.

The dissimilar structure of the ice matrices can account for the different spectral observations (Gálvez et al. 2010). In compact $\mathrm{HQ}$ ices the $\mathrm{OCN}^{-}$ions are surrounded by water molecules, and when the temperature is raised and the water molecules rearrange to form their crystalline structure, the ions cannot break their environment and diffuse in the ice to meet their counter ions. The result is that the $\mathrm{OCN}^{-}$ions are still in an environment similar to what they had at a lower temperature. Only when water ice sublimates does the ionic species form the KOCN solid and regain infrared intensities (lower traces in Figure 4). By contrast, for vapor-deposited ices as in the experiment of van Broekhuizen et al. (2004), the neutral species are embedded in a porous amorphous water ice structure which, when warmed, starts to collapse giving the molecules the possibility to diffuse within the ice and react to form the ionic species. In particular, $\mathrm{HNCO}$ and $\mathrm{NH}_{3}$ will find each other and a proton transfer can take place to form $\mathrm{OCN}^{-}$and $\mathrm{NH}_{4}{ }^{+}$, which will then remain adjacent in segregated ionic networks, in which the cyanate vibrations give higher infrared absorption intensities, comparable to those of the solid residue after water evaporation (Gálvez et al. 2010). This different environment is also responsible for the dissimilar IR-band profiles, which are more symmetrical in the porous ices.

\section{ASTROPHYSICAL IMPLICATIONS}

The XCN band observed in icy mantles on interstellar grain surfaces within molecular clouds is of great interest since it can be taken as a tracer for the chemical evolution associated with the process of star formation. Astrophysical observations and laboratory data for this band do not always match. The present work presents new data on the IR spectral bands of $\mathrm{OCN}^{-}$ ions in astronomical ice analogs. In contrast to previous studies, which are based on the energetic processing of initially porous vapor-deposited ice mixtures (see van Broekhuizen et al. 2005 and references therein), the preparation procedure (HQ) chosen in our experiments ensures that the $\mathrm{OCN}^{-}$ions are in close interaction with the surrounding water molecules in compact ice samples. This is in principle a realistic astronomical environment that, as far as we know, has not been investigated before. A significant weakening and the appearance of a secondary peak in the $4.62 \mu \mathrm{m}$ absorption feature of the $\mathrm{OCN}^{-}$ion are the main novelties with respect to the previous laboratory studies. This marked attenuation of the IR bands in the HQ compact ices as compared with those from processed porous samples was also observed in previous works by our group on $\mathrm{NH}_{4}{ }^{+}$and on $\mathrm{HCOO}^{-}$ions. The results of our experiments suggested that in the course of processing the porous samples, ionic networks rather than solvated isolated ions could segregate within the ice (Gálvez et al. 2010). Both situations could be found in interstellar environments, and consequently, the present measurements and those from previous works on $\mathrm{OCN}^{-}$should be regarded as complementary.

In our experiments $\mathrm{OCN}^{-}$and water are the only molecules present in the samples, and the band shows a double peak structure. In the majority of laboratory studies, where cyanate has 


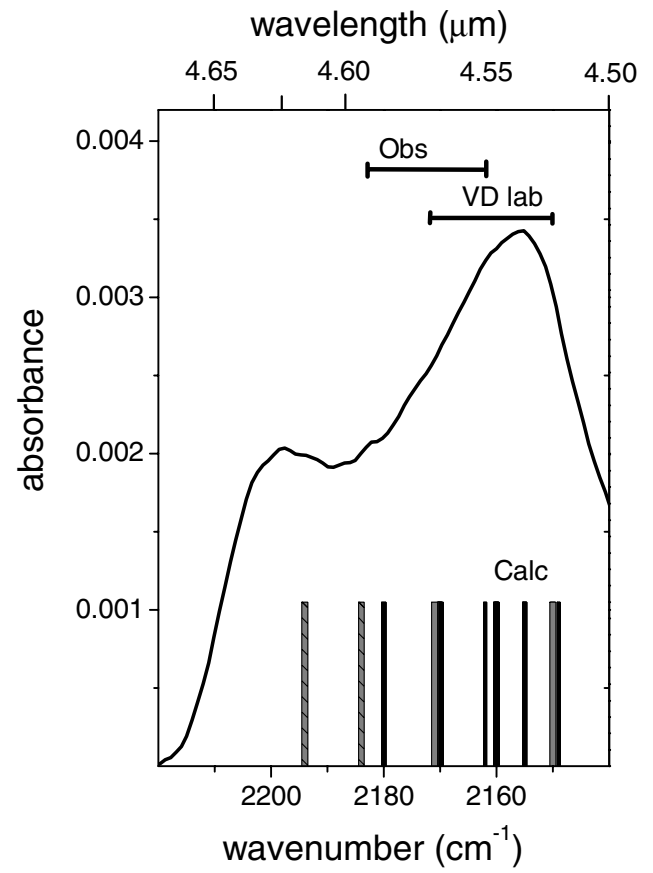

Figure 5. Band profile of the $\mathrm{CN}$ stretch absorption, enlarged from panel (a) of Figure 3. Vertical bars indicate calculated frequencies for this band. Black: $\mathrm{OCN}^{-}$in ice networks (SIESTA, this work); grey: cyanate-water dimers (Gaussian, this work); hatched: $\mathrm{OCN}^{-} .\left(\mathrm{H}_{2} \mathrm{O}\right)_{15}$ aggregates (Park \& Woon 2004). The horizontal lines span frequency ranges of values, taken from van Broekhuizen et al. (2005), for the XCN band observed in different astrophysical objects (Obs), and for the $\nu_{3}$ band of $\mathrm{OCN}^{-}$in a variety of laboratory experiments on vapor-deposited ices (VD lab).

been synthesized by irradiation of more or less complex mixtures, other molecular species are created as well, complicating the analysis of the spectra. In addition to this, the concentration of the initial sample and the type (UV or ion bombardment) and extent of irradiation can alter the outcome of the experiment (see the compilation by van Broekhuizen et al. 2005). In astrophysical media, where the generation mechanism of $\mathrm{OCN}^{-}$is still debated, other molecules will surely be present as well, but the understanding of the spectra can be facilitated if individual features are unambiguously tagged to begin with.

We have represented in Figure 5 the band profile of the $\mathrm{CN}$ stretch recorded in our laboratory, indicating by two horizontal lines at the top the spreading in frequency position observed for the XCN band in different astrophysical objects (Obs) or measured in various laboratory experiments (VD lab). It can be seen that the laboratory data fall within our lower frequency peak. It has been stated in the literature that this band is strongly affected by the environment, and that different chemical surroundings would be needed to explain the spread in frequencies of the astronomical observations (Öberg et al. 2011). In this work we prove that a simple amorphous water surrounding could be enough to displace the frequency position of the band toward the high-frequency region where the astrophysical observations fall. This experimental evidence is supported by calculations. The vertical bars represented in the lower part of Figure 5 indicate the predicted values of the $\mathrm{CN}$ stretch of $\mathrm{OCN}^{-}$estimated in this work and in a previous calculation by Park \& Woon (2004). The theoretical values, obtained by modeling the interaction of $\mathrm{OCN}^{-}$ions with water under different approximations, predict a large frequency spreading that falls within our experimental profile in all cases.
Table 2

Estimated Attenuation Factors and Corrected Infrared Band Strengths for the Indicated Spectral Regions, Based on the Solid Residue to HQ Deposit Approximate Intensity Ratios

\begin{tabular}{lccc}
\hline \hline Species & $\begin{array}{c}\text { Spectral Region } \\
\left(\mathrm{cm}^{-1}\right)\end{array}$ & $\begin{array}{c}\text { Attenuation } \\
\text { Factor }\end{array}$ & $\begin{array}{c}A^{\mathrm{a}} \\
\left(\mathrm{cm} \mathrm{mol}^{-1}\right)\end{array}$ \\
\hline $\mathrm{NH}_{4}{ }^{+}$ & $1400-1500$ & 28 & $1.57 \times 10^{-18}$ \\
$\mathrm{HCOO}^{-}$ & $1300-1400$ & 2.7 & $1.44 \times 10^{-17}$ \\
$\mathrm{OCN}^{-}$ & $2200-2100$ & 15 & $8.66 \times 10^{-18}$ \\
\hline
\end{tabular}

Note. ${ }^{a}$ Corrected A's from the reference values given in Schutte \& Khanna (2003), Gálvez et al. (2010), and van Broekhuizen et al. (2004), respectively.

Another practical result of interest is the estimation of the apparent weakening of the CN stretching band in HQ compact samples. We have drawn a tentative background contour of the spectral regions of interest, taking into account the ice water bands when necessary, and used it to make a rough estimation of the apparent intensity depletion of the spectra of the ions in compact ice matrices as compared to the corresponding bands in the evaporated samples. We show in Table 2 the result of this estimation for the species studied so far (Maté et al. 2009; Gálvez et al. 2010), based on experiments of similar ion concentrations, $5 \%-7 \%$, and for the specific regions indicated in the table. As discussed above, we assume that the intensity of bands in the solid salt deposit is roughly the same as that in the ionic networks where they are located after acid-base chemistry in porous ice mixtures. With this postulation, it is possible to apply the attenuation factor to calculate corrected infrared strengths, $A$, for these bands when the ions are embedded in a compact amorphous water environment. In the last column of Table 2 we present corrected $A$ values obtained from the ones given by Schutte \& Khanna (2003), Gálvez et al. (2010), and van Broekhuizen et al. (2004) for $\mathrm{NH}_{4}{ }^{+}, \mathrm{HCOO}^{-}$and $\mathrm{OCN}^{-}$, respectively. The figures in Table 2 indicate that the region of the $v_{4}$ band of $\mathrm{NH}_{4}{ }^{+}$suffers a very large broadening that would hamper ammonium recognition in the presence of compact amorphous water, whereas for $\mathrm{HCOO}^{-}$the selected spectral zone is a good candidate for the observation of this anion; $\mathrm{OCN}^{-}$is in an intermediate situation, where the chosen band, the best option for astrophysical detection, undergoes a considerable broadening and apparent weakening, but can still indicate the presence of the ions in compact water abundant media, although their observation would be much favored in compact water scarce systems.

This research has been carried out with funding from the Spanish Ministry of Education, Project FIS2010-16455, and within the frame of the Unidad Asociada UCM-CSIC. Y.R.-L. acknowledges financial support from CSIC, JAE-Doc Program. Part of the computations has been carried out at CESGA, FinisTerrae (Spain). P.C.G. gratefully acknowledges financial support from the Spanish Ministry of Science and Innovation, Project CTQ-2008-02578/BQU.

\section{REFERENCES}

Abad, L., Bermejo, D., Herrero, V. J., Santos, J., \& Tanarro, I. 1995, Rev. Sci. Instrum., 66, 3826

Accolla, M., Congiu, E., Dulieu, F., et al. 2011, PCCP, 13, 8037

Baragiola, R. A. 2003, in Water in Confining Geometries, Microporous Amorphous Water Ice Thin Films: Properties and Their Astronomical Implications, ed. V. Buch \& J. P. Devlin (Berlin: Springer)

Bernstein, M. P., Sandford, S. A., \& Allamandola, L. J. 2000, ApJ, 542, 894 
Demyk, K., Dartois, E., d'Hedecourt, L., et al. 1998, A\&A, 339, 553

Fleissner, G., Hallbrucker, A., \& Mayer, E. 1995, J. Chem. Phys., 99, 8401

Gálvez, O., Maté, B., Herrero, V. J., \& Escribano, R. 2008, Icarus, 197, 599

Gálvez, O., Maté, B., Herrero, V. J., \& Escribano, R. 2010, ApJ, 724, 539

Frisch, M. J., Trucks, G. W., Schlegel, H. B., et al. 2004, Gaussian 03, Revision C.02 (Wallingford, CT: Gaussian, Inc.)

Gibb, E. L., Whittet, D. C. B., Schutte, W. A., et al. 2000, ApJ, 536, 347

Grim, R. J. A., \& Greenberg, J. M. 1987, ApJ, 321, L91

Hudson, E. L., Moore, M. H., \& Gerakines, P. A. 2001, ApJ, 550, 1140

Johari, G. P., Hallbrucker, A., \& Mayer, E. 1987, Nature, 330, 552

Martín-Llorente, B., Fernández-Torre, D., Herrero, V. J., et al. 2006, Chem. Phys. Lett., 427, 300

Mastrapa, R. M., Shandford, S. A., Roush, T. L., et al. 2009, ApJ, 701,1347

Maté, B., Medialdea, A., Moreno, M. A., Escribano, R., \& Herrero, V. J. 2003, J. Phys. Chem. B, 107, 11098

Maté, B., Gálvez, O., Herrero, V. J., et al. 2009, ApJ, 703, L178

Maté, B., Rodríguez-Lazcano, Y., \& Herrero, V. J. 2012, PCCP, 14, 10595

Mayer, E. 1985, J. Appl. Phys., 58, 663

Mispelaer, F., Theule, P., Duvernay, F., et al. 2012, A\&A, 540, A40

Novozamsky, J. H., Schutte, W. A., \& Keane, J. V. 2001, A\&A, 379, 588

Oba, Y., Miyauchi, N., Hidaka, H., et al. 2009, ApJ, 701, 464
Öberg, K. I., Boogert, A. C. A., Pontoppidan, K. M., et al. 2011, ApJ, 740, 109 Ordejón, P., Artacho, E., \& Soler, J. M. 1996, Phys. Rev. B, 53, R10441 Palumbo, M. E., Pendleton, Y. J., \& Strazzulla, G. 2000, ApJ, 542, 890 Palumbo, M. E. 2006, A\&A, 453, 903

Park, J.-Y., \& Woon, D. E. 2004, J. Phys. Chem. A, 108, 6589

Pendleton, Y. J., Tielens, A. G. G. M., Tokunaga, A. T., \& Bernstein, M. P. 1999, ApJ, 513, 294

Pontoppidan, K. M., Fraser, H. J., Dartois, E., et al. 2003, A\&A, 408, 981

Raunier, S., Chiavassa, T., Marinelli, F., et al. 2003, J. Phys. Chem. A, 107, 9335

Romanzin, C., Ioppolo, S., Cuppen, H. M., van Dishoeck, E. F., \& Linnartz, H. 2011, J. Chem. Phys., 134, 084504

Schutte, W. A., \& Greenberg, J. M. 1997, A\&A, 317, L43

Schutte, W. A., \& Khanna, R. K. 2003, A\&A, 398, 1049

Soifer, B. T., Puetter, R. C., Russell, R. W., et al. 1979, ApJ, 232, L53

Soler, J. M., Artacho, E., Gale, J. D., et al. 2002, J. Phys.: Condens. Matter, 14, 2745

Theule, P., Duvernay, F., Ilmane, A., et al. 2011, A\&A, 530, A96

van Broekhuizen, F. A., Keane, J. V., \& Schutte, W. A. 2004, A\&A, 415, 425

van Broekhuizen, F. A., Pontoppidan, K. M., Fraser, K. M., \& van Dishoeck, E. F. 2005, A\&A, 441, 249

Whittet, D. C. B., Pendleton, Y. J., Gibb, E. L., et al. 2001, ApJ, 550, 793 